\title{
ONE-PARAMETER FAMILIES OF CURVES IN THE PLANE*
}

GABRIEL M. GREEN

\section{INTRODUCTION}

Nets of curves in the plane were studied from a projective point of view for the first time by Wilczynski. $\dagger$ As he himself points out, $\ddagger$ the theory as set up by him is that of the "two component one-parameter families, rather than a theory of the net itself." In the present paper, we shall show how a single one-parameter family of plane curves may be studied by Wilczynski's methods. Although many of the projective properties of a single one-parameter family may be investigated by considering it as a component family of a particular net,- - such properties are in fact noted in Wilczynski's memoirstill the theory of one of the families of the net is closely bound up with the properties of the other, if the study be made as in the paper already cited. To make this clearer, we must outline Wilczynski's procedure.

If $y^{(1)}, y^{(2)}, y^{(3)}$ be interpreted as the homogeneous coördinates of a point in the plane, the equations

$$
y^{(k)}=f^{(k)}(u, v)
$$$$
(k=1,2,3)
$$

define for $u=$ const. and $v=$ const. two one-parameter families of curves. Equations (1) are a fundamental system of solutions of the completely integrable system of partial differential equations

$$
\begin{aligned}
& y_{u u}=a y_{u}+b y_{v}+c y, \\
& y_{u v}=a^{\prime} y_{u}+b^{\prime} y_{v}+c^{\prime} y, \\
& y_{v v}=a^{\prime \prime} y_{u}+b^{\prime \prime} y_{v}+c^{\prime \prime} y,
\end{aligned}
$$

any fundamental system of solutions of which, say $\bar{y}^{(1)}, \bar{y}^{(2)}, \bar{y}^{(3)}$, will be of the form

$$
\bar{y}^{(k)}=c_{k 1} y^{(1)}+c_{k 2} y^{(2)}+c_{k 3} y^{(3)}, \quad\left|c_{k l}\right| \neq 0 \quad(k=1,2,3)
$$

where the $c$ 's are constants. The differential equations therefore define the

* Presented to the Society October 25, 1913.

† One-parameter families and nets of plane curves. These Transactions, vol. 12 (1911), pp. 473-510.

$\ddagger$ Loc. cit., p. 478. 
configuration (1) and all its projective transformations. The most general point transformations which leave unchanged the configuration (1) are composed of transformations of the form

$$
\bar{y}=\lambda(u, v) y,
$$

which leave every point of the plane fixed, and

$$
\bar{u}=U(u), \quad \bar{v}=V(v),
$$

which leave invariant each of the families $u=$ const., $v=$ const. Suppose these transformations to be made on the differential equations (2). Those properties of (2) which remain unchanged under these transformations will be characteristic of the net (1) and of all its projective transformations, $i$. e., they will be projective properties. Invariants are those functions of the coefficients of (2), and of their derivatives, which remain unchanged under these transformations, except possibly for a factor depending only on the transformations. Covariants have the same nature and properties, but contain also the variable $y$ and its derivatives.

Now evidently the projective properties of the net, as expressed by the vanishing of invariants thus found, can not all be characteristic of a single family of curves of the net, say the family $u=$ const. For instance, the condition that the curves $v=$ const. be straight lines is obviously from the first of equations (2) the vanishing of the invariant $b$; this has, however, nothing whatever to do with the properties of the curves $u=$ const. But the invariant equation $a^{\prime \prime}=0$ is the condition that the curves $u=$ const. be straight lines, and is therefore characteristic for that family.

Again, the covariants of the net have the following geometric interpretation. The tangents to the curves of one family, constructed at the points where these curves meet a fixed curve of the other family, have a covariant curve as envelope. Evidently neither of the two covariants thus obtained is intrinsically connected with the single family of curves $u=$ const.

The theory of the net of curves, as developed by Wilczynski, is therefore not applicable in its present form to the study of a single family of plane curves, say the curves $u=$ const. defined by (1). We shall in the sequel modify his theory so that it may be used for the theory of a one-parameter family of curves, by making this family one of the components of a net, in which the second family bears an intrinsic geometric relation to the given family.

\section{The One-parameter Family of Curves as Part of a Canonical Net}

The most general transformation of the independent variables which leaves fixed the family of curves $u=$ const. defined by equations (1) is 


$$
\bar{u}=U(u), \quad \bar{v}=V(u, v) .
$$

By means of this transformation we can change in the most arbitrary way the system of curves $v=$ const. which cut the curves $u=$ const. We seek a particular transformation of form (3) which will associate with the family $u=$ const. a family $v=$ const. intrinsically related to it. For a family of space curves which do not lie on a developable surface and which are not asymptotic curves on the surface determined by them, there is a very evident choice of a second family, namely the conjugate system of curves.* For the system of plane curves there are of course isogonal trajectories, but isogonality is not a projective property. There is in fact no obvious projective relation, analogous say to the relation of conjugacy of curves on a surface, of which we can make use here. We proceed to determine a family of curves $v=$ const. which have a projectively intrinsic relation to the given family $u=$ const., but the relation is by no means so simple as that of conjugacy on a curved surface.

Let us carry out the transformation

$$
\bar{u}=u, \quad \bar{v}=\phi(u, v),
$$

which is included in (3), on the system of differential equations. We have

$$
\begin{aligned}
y_{u} & =\bar{y}_{u}+\bar{y}_{v} \phi_{u}, \quad y_{v}=\bar{y}_{v} \phi_{v}, \\
y_{u u} & =\bar{y}_{u u}+2 \bar{y}_{u v} \phi_{u}+\bar{y}_{v v} \phi_{u}^{2}+\bar{y}_{u}+\bar{y}_{v} \phi_{u u}, \\
y_{u v} & =\bar{y}_{u v} \phi_{v}+\bar{y}_{v v} \phi_{u} \phi_{v}+\bar{y}_{v} \phi_{u v}, \\
y_{v v} & =\bar{y}_{v v} \phi_{v}^{2}+\bar{y}_{v} \phi_{v v},
\end{aligned}
$$

where we have written $\bar{y}_{u}$ for $\partial y / \partial \bar{u}, \bar{y}_{v}$ for $\partial y / \partial \bar{v}$, etc. Substituting these equations in (2), and solving for the new second derivatives $\bar{y}_{u u}, \bar{y}_{u v}, \bar{y}_{v v}$, we obtain a system of differential equations of the same form as (2), with coefficients $\bar{a}, \bar{b}$, etc., given by

(6)

$$
\begin{aligned}
\bar{a}=a-2 a^{\prime} \frac{\phi_{u}}{\phi_{v}}+a^{\prime \prime} \frac{\phi_{u}^{2}}{\phi_{v}^{2}}, & \\
\bar{b}=\left(a-2 b^{\prime}\right) \phi_{u}+b \phi_{v}+\left(b^{\prime \prime}-2 a^{\prime}\right) \frac{\phi_{u}^{2}}{\phi_{v}} & +a^{\prime \prime} \frac{\phi_{u}^{3}}{\phi_{v}^{2}} \\
& -\phi_{u u}+2 \frac{\phi_{u}}{\phi_{v}} \phi_{u v}-\frac{\phi_{v}^{2}}{\phi_{v}^{2}} \phi_{v v},
\end{aligned}
$$

$$
\bar{c}=c-2 c^{\prime} \frac{\phi_{u}}{\phi_{v}}+c^{\prime \prime} \frac{\phi_{u}^{2}}{\phi_{v}^{2}}
$$

$$
\phi_{v} \bar{a}^{\prime}=a^{\prime}-a^{\prime \prime} \frac{\phi_{u}}{\phi_{v}}, \quad \phi_{v} \bar{c}^{\prime}=c^{\prime}-c^{\prime \prime} \frac{\phi_{u}}{\phi_{v}},
$$

* The author has already carried out this idea in a paper entitled Projective Differential Geometry of One-parameter Families of Space Curves, and Conjugale Nets on a Curved Surface, to appear in the American Journal of Mathematics. 


$$
\begin{aligned}
\bar{b}^{\prime} & =b^{\prime}+\left(a^{\prime}-b^{\prime \prime}\right) \frac{\phi_{u}}{\phi_{v}}-a^{\prime \prime} \frac{\phi_{u}^{2}}{\phi_{v}^{2}}-\frac{\phi_{u v}}{\phi_{v}}+\frac{\phi_{u}}{\phi_{v}} \frac{\phi_{v v}}{\phi_{v}}, \\
\phi_{v}^{2} \bar{a}^{\prime \prime} & =a^{\prime \prime}, \quad \phi_{v} \bar{b}^{\prime \prime}=b^{\prime \prime}+a^{\prime \prime} \frac{\phi_{u}}{\phi_{v}}-\frac{\phi_{v v}}{\phi_{v}}, \quad \phi_{v}^{2} \bar{c}^{\prime \prime}=c^{\prime \prime} .
\end{aligned}
$$

Let us put

$$
\mathfrak{A}^{\prime}=a^{\prime}-\frac{1}{3}\left(a^{\prime}+b^{\prime \prime}\right)+\frac{1}{6} \frac{a_{0}^{\prime \prime}}{a^{\prime \prime}},
$$

and calculate the corresponding quantity $\overline{\mathfrak{P}}^{\prime}$. We have

and

$$
\frac{\bar{a}_{v}^{\prime \prime}}{\bar{a}^{\prime \prime}}=\frac{\partial}{\partial \bar{v}} \log \bar{a}^{\prime \prime}=\frac{1}{\phi_{v}} \frac{\partial}{\partial v} \log \bar{a}^{\prime \prime}=\frac{1}{\phi_{v}}\left(\frac{a_{v}^{\prime \prime}}{a^{\prime \prime}}-2 \frac{\phi_{v v}}{\phi_{v}}\right),
$$

$$
\begin{aligned}
\phi_{v} \overline{\mathfrak{A}}^{\prime} & =\phi_{v}\left[\bar{a}^{\prime}-\frac{1}{3}\left(\bar{a}^{\prime}+\bar{b}^{\prime \prime}\right)+\frac{1}{6} \frac{\bar{a}_{v}^{\prime \prime}}{\bar{a}^{\prime \prime}}\right] \\
& =a^{\prime}-\frac{1}{3}\left(a^{\prime}+b^{\prime \prime}\right)+\frac{1}{6} \frac{a_{v}^{\prime \prime}}{a^{\prime \prime}}-a^{\prime \prime} \frac{\phi_{u}}{\phi_{v}} \\
& =\mathscr{P}^{\prime}-a^{\prime \prime} \frac{\phi_{u}}{\phi_{v}} .
\end{aligned}
$$

We may therefore make $\overline{\mathfrak{A}}^{\prime}$ vanish by taking for $\phi$ a solution of the partial differential equation of the first order

$$
a^{\prime \prime} \phi_{u}-\mathfrak{Y}^{\prime} \phi_{v}=0 .
$$

This choice of $\phi(u, v)$ gives a uniquely determined family $\bar{v}=$ const. For, from (7) we see that the most general transformation of the independent variable $v$ which leaves the condition $\mathfrak{Q}^{\prime}=0$ unchanged is independent of $u$; also, the condition will be found to remain invariant under any transformation $\bar{u}=U(u)$. Consequently the family $\ddot{v}=$ const. bears a characteristic relation to the family $\bar{u}=$ const., of such nature, that it remains undisturbed only by those transformations of the independent variables which are of the form

$$
\bar{u}=U(\bar{u}), \quad \bar{v}=V(\bar{v}) .
$$

We can make this clearer by inquiring into the geometric meaning of the relation $\mathfrak{A}^{\prime}=0$. This condition has been interpreted by Wilczynski.* We shall give here an adequate description of the geometric nature of the net, but must refer to Wilczynski's memoir for the demonstrations.

Consider any net $u=$ const., $v=$ const. At each point of a fixed curve $u=$ const. construct the tangent to the curve $v=$ const. which passes through that point. The $\infty^{1}$ tangents so drawn will envelop a curve. The family of

* Loc. cit., p. 504. 
curves corresponding in this way to the family $u=$ const. is given by the equations

$$
\rho^{(k)}=y_{k}^{(k)}-b^{\prime} y^{(k)} \quad(k=1,2,3),
$$

or, as we may say, by the covariant (of the net)

$$
\rho=y_{u}-b^{\prime} y,
$$

for $u=$ const. ${ }^{*}$ Thus to every point $P_{y}$ given by the equations

$$
y^{(k)}=f^{(k)}(u, v)
$$

corresponds a covariant point $P_{p}$. In the same way, a covariant

$$
\sigma=y_{v}-a^{\prime} y
$$

gives points $P_{\sigma}$, which for $v=$ const. trace the envelope of the tangents to the curves $u=$ const. at the points where these curves meet the fixed curve $v=$ const. given by $y$. We have then at any point $P_{y}$ two lines, tangent respectively to the curves $u=$ const. and $v=$ const. which pass through $P_{y}$, and on these two lines the respective covariant points $P_{\sigma}, P_{\rho}$.

Now construct the conic which osculates the curve $u=$ const. at $P_{y}$. The line $P_{\sigma} P_{\rho}$ cuts it in a pair of points, which are harmonic to the pair $P_{\rho}, P_{\sigma}$ 'f and only if $\mathfrak{R}^{\prime}=0 . \dagger$

Let us call the net so determined the canonical net. The covariants

$$
\bar{\rho}=\bar{y}_{u}-\bar{b}^{\prime} \bar{y}, \quad \bar{\sigma}=\bar{y}_{v}-\bar{a}^{\prime} \bar{y}
$$

will give for each point $P_{y}$ two points $P_{\bar{\rho}}$ and $P_{\ddot{\sigma}}$, and the line $P_{y} P_{\bar{\rho}}$ will be the polar of $P_{\bar{\sigma}}$ with respect to the conic which osculates the curve $u=$ const. at $P_{y}$. We proceed now to show geometrically how the family $u=$ const. determines the second family, $\bar{v}=$ const., of the canonical net. Of course, the best we can do is to find at each point $P_{y}$ the direction of the line $P_{y} P_{\bar{p}}$, since the determination of the family $\bar{v}=$ const. itself requires the integration of equation (8).

Suppose known only the family of curves $u=$ const. Draw the tangent $t$ and the osculating conic $C$ at $P_{y}$. Consider any line $l$ through $P_{y}$. Its pole with respect to the conic $C$ is a point $Q$ on $t$. The tangents to the curves $u=$ const. at the points where these curves meet $l$ envelop a curve; let $R$ be the point of this curve which lies on $t$. Consider now the pencil of lines $[l]$ through $P_{y}$. The corresponding poles will be a range $[Q]$ on $t$; corresponding to each $Q$ will be a point $R$ given by the construction above. The ranges $[Q]$ and $[R]$ are in one to one correspondence. There are two double-points $Q \equiv R$; one of them is the point $P_{y}$, the other a definite point which is in

*. J. Wilczynski, loc. cit., p. 486.

† E. J. Wilczynski, loc. cit., p. 504. 
fact the covariant point $P_{\bar{\sigma}}$. The polar of $P_{\bar{\sigma}}$ with respect to $C$ gives a certain direction passing through $P_{y}$. This will give the direction of $P_{y} P_{\bar{p}}$, although the point $P_{\bar{\rho}}$ is not thereby determined. It is possible, therefore, to construct at each point $P_{y}$ the direction $P_{y} P_{\bar{p}}$; the $\infty^{2}$ directions so constructed give on integration the second family, $\bar{v}=$ const., of the canonical net.

Since the canonical net is completely determined by the given family of curves $u=$ const., it follows that the invariants and covariants of the net are invariants and covariants of the given family of curves. Thus the quantities $\bar{\rho}, \bar{\sigma}$ given by (9) are covariants of the family $u=$ const. The invariants may be calculated as in Wilczynski's memoir. Put

$$
\begin{gathered}
\bar{f}_{u}=\bar{a}+\bar{b}^{\prime}, \quad \bar{f}_{v}=\bar{a}^{\prime}+\bar{b}^{\prime \prime}, \\
\bar{A}=\bar{a}-\frac{2}{3} \bar{f}_{u}, \quad \bar{B}=\bar{b}, \quad \bar{C}=\bar{c}+\frac{1}{3} \bar{a}_{u}+\frac{1}{3} \bar{b} \bar{f}_{v}-\frac{1}{3} \bar{f}_{u u}-\frac{1}{8} \bar{f}_{u}^{2}, \\
\bar{A}^{\prime}=\bar{a}^{\prime}-\frac{1}{3} \bar{f}_{v}, \quad \bar{B}^{\prime}=\bar{b}^{\prime}-\frac{1}{3} \bar{f}_{u}, \\
\bar{C}^{\prime}=\bar{c}^{\prime}+\frac{1}{3} \bar{a}^{\prime} \bar{f}_{u}+\frac{1}{3} \bar{b}^{\prime} \bar{f}_{v}-\frac{1}{3} \bar{f}_{u v}-\frac{1}{8} \bar{f}_{u} \bar{f}_{v}, \\
\bar{A}^{\prime \prime}=\bar{a}^{\prime \prime}, \quad \bar{B}^{\prime \prime}=\bar{b}^{\prime \prime}-\frac{2}{3} \bar{f}_{v}, \\
\bar{C}^{\prime \prime}=\bar{c}^{\prime \prime}+\frac{1}{3} \bar{a}^{\prime \prime} \bar{f}_{u}+\frac{1}{3} \bar{b}^{\prime \prime} \bar{f}_{v}-\frac{1}{3} \bar{f}_{v v}-\frac{1}{8} \bar{f}_{v}^{2} .
\end{gathered}
$$

Then every invariant of the canonical net, and hence of the family of curves $u=$ const., is a function of the following fundamental system of invariants and their derivatives:*

$$
\begin{aligned}
& \overline{\mathfrak{B}}=\bar{B}, \quad \overline{\mathfrak{C}}=\bar{C}-\bar{B}_{u}^{\prime}-2 \bar{B}^{2}+\bar{A}^{\prime} \bar{B}, \\
& \overline{\mathfrak{A}}^{\prime}=\bar{A}^{\prime}+\frac{1}{6} \frac{\overline{A_{v}^{\prime \prime}}}{\bar{A}^{\prime \prime}}, \quad \overline{\mathfrak{B}}^{\prime}=\bar{B}^{\prime}+\frac{1}{6} \frac{\overline{B_{u}}}{\bar{B}}, \quad \overline{\mathfrak{C}}^{\prime}=\bar{C}^{\prime}+\bar{A}^{\prime} \bar{B}^{\prime}, \\
& \overline{\mathfrak{A}}^{\prime \prime}=\bar{A}^{\prime \prime}, \quad \overline{\mathfrak{C}^{\prime \prime}}=\bar{C}^{\prime \prime}-\bar{A}_{v}^{\prime}-2 \bar{A}^{\prime 2}+\bar{A}^{\prime \prime} \bar{B}^{\prime} .
\end{aligned}
$$

The last statement is evidently true from a geometric point of view; analytically, it may be verified as follows. In equations (6) appear the first and second derivatives of $\phi$. From (8) we obtain by differentiation the equations

$$
\begin{aligned}
& \phi_{u u}-\frac{\phi_{u}}{\phi_{v}} \phi_{u v}=\frac{\mathfrak{R}_{u}^{\prime}}{a^{\prime \prime}} \phi_{v}-\frac{a_{u}^{\prime \prime}}{a^{\prime \prime}} \phi_{u}, \\
& \phi_{u v}-\frac{\phi_{u}}{\phi_{v}} \phi_{v v}=\frac{\mathfrak{R}_{v}^{\prime}}{a^{\prime \prime}} \phi_{v}-\frac{a_{v}^{\prime \prime}}{a^{\prime \prime}} \phi_{u} .
\end{aligned}
$$

By means of these and (8) we may eliminate from expressions (6) all derivatives of $\phi$ but one, say $\phi_{v}$, except in the expression for $\bar{b}^{\prime \prime}$, from which $\phi_{v}$ and $\phi_{v v}$ cannot be removed. In all of the expressions thus obtained $\phi_{v}$ comes

\footnotetext{
* The invariant $\overline{\mathcal{Q}^{\prime}}=\bar{A}^{\prime}+\frac{1}{6} \frac{\bar{A}_{v}^{\prime \prime}}{\overline{A^{\prime \prime}}}$ is of course identically equal to zero.
} 
out as a factor; moreover, in the invariants (12) $\bar{b}^{\prime \prime}$ occurs only in combinations from which $\phi_{v v}$ disappears. It follows upon calculation that except for extraneous factors (powers of $\phi_{v}$ ), the invariants (12) are expressible rationally in terms of the coefficients of the original system of differential equations (2), and the derivatives of these coefficients.

The covariants (9) are expressible in the same way; we have, in fact,

$$
\bar{y}_{u}=y_{u}-\frac{\phi_{u}}{\phi_{v}} y_{v}, \quad \bar{y}_{v}=\frac{1}{\phi_{v}} y_{v},
$$

so that on calculating the values of $\bar{a}^{\prime}$ and $\bar{b}^{\prime}$ we obtain

$$
\begin{aligned}
\bar{\rho} & =y_{u}-\frac{\mathfrak{U}^{\prime}}{a^{\prime \prime}} y_{v}-\left[b^{\prime}+\frac{\mathfrak{U}^{\prime}}{a^{\prime \prime}}\left(a^{\prime}-b^{\prime \prime}-\mathfrak{U}^{\prime}+\frac{a_{v}^{\prime \prime}}{a^{\prime \prime}}-\frac{\mathfrak{X}_{v}^{\prime}}{\mathfrak{X}^{\prime}}\right)\right] y, \\
\phi_{v} \bar{\sigma} & =y_{v}-\left(a^{\prime}-\mathfrak{U}^{\prime}\right) y .
\end{aligned}
$$

All covariants of the canonical net are functions of these, of $y$, and of invariants; it may be shown, moreover, that the complete system of invariants, as set up by Wilczynski, is expressible entirely in terms of the coefficients of (2). We may state our results as follows: the projective geometry of a oneparameter family of plane curves may be investigated by the study of a canonical net whose determination requires the integration of a partial differential equation of the first order. This integration is however unnecessary for the formulation of the projective properties of the one-parameter family of curves, since the invariants and covariants of this geometric configuration are expressible entirely in terms of the coefficients and variables of the original system of differential equations which define the one-parameter family as one component family of a net in which the second component family is arbitrary.

\section{Canonical Development for a One-parameter Family of Prane Curves}

Suppose the family $v=$ const. has been determined so that it forms with the given system $u=$ const. a canonical net. We have then

$$
\begin{gathered}
\bar{y}_{u}=\bar{\rho}+\bar{b}^{\prime} \bar{y}, \quad \bar{y}_{v}=\bar{\sigma}+\bar{a}^{\prime} \bar{y}, \\
\bar{y}_{u u}=\bar{a} \bar{y}_{u}+\bar{b} \bar{y}_{v}+\bar{c} \bar{y}, \\
\bar{y}_{u v}=\bar{a}^{\prime} y+\bar{b}^{\prime} \bar{y}_{v}+\bar{c}^{\prime} \bar{y}, \\
\bar{y}_{v v}=\bar{a}^{\prime \prime} \bar{y}_{u}+\bar{b}^{\prime \prime} \bar{y}_{v}+\bar{c}^{\prime \prime} \bar{y} .
\end{gathered}
$$

The second derivatives of $\bar{y}$ are therefore expressible linearly in terms of $\bar{\rho}, \bar{\sigma}, \bar{y}$; the same is easily seen to be true of all higher derivatives of $\bar{y}$. We have, then, in general, 


$$
\frac{\partial^{i+j}}{\partial \bar{u}^{i} \partial \bar{v}^{j}} \bar{y}=\alpha^{(i j)} \bar{\rho}+\beta^{(i j)} \bar{\sigma}+\gamma^{(i j)} \bar{y}
$$

where the $\alpha$ 's, $\beta$ 's, and $\gamma$ 's are functions of the coefficients in (15b) and of their derivatives. Thus

$$
\begin{array}{lll}
\alpha^{(10)}=1, & \beta^{(10)}=0, & \gamma^{(10)}=\bar{b}^{\prime}, \\
\alpha^{(01)}=0, & \beta^{(01)}=1, & \gamma^{(01)}=\bar{a}^{\prime},
\end{array}
$$

from (15a). We may call these "coefficients of the first order." The coefficients of the second order are

$$
\begin{array}{lll}
\alpha^{(20)}=\bar{a}, & \beta^{(20)}=\bar{b}, & \gamma^{(20)}=\bar{c}+\bar{a} \bar{b}^{\prime}+\bar{b} \bar{a}^{\prime}, \\
\alpha^{(11)}=\bar{a}^{\prime}, & \beta^{(11)}=\bar{b}^{\prime}, & \gamma^{(11)}=\bar{c}^{\prime}+2 \bar{a}^{\prime} \bar{b}^{\prime}, \\
\alpha^{(02)}=\bar{a}^{\prime \prime}, & \beta^{(02)}=\bar{b}^{\prime \prime}, & \gamma^{(02)}=\bar{c}^{\prime \prime}+\bar{a}^{\prime \prime} \bar{b}^{\prime}+\bar{b}^{\prime \prime} \bar{a}^{\prime} .
\end{array}
$$

Some of the coefficients of the third and higher orders are expressible in more than one way; this results from the fact that an expression like

$$
\alpha^{(21)} \bar{\rho}+\beta^{(21)} \bar{\sigma}+\gamma^{(21)} \bar{y}
$$

may be found either from $\partial \bar{y}_{u u} / \partial \bar{v}$ or from $\partial \bar{y}_{u v} / \partial \bar{u}$. We suppose that in every case two or more expressions for the same coefficient are identically equal* (for all values of $\bar{u}$ and $\bar{v}$ ). Thus, we have

$$
\alpha^{(21)}=\bar{a}_{u}^{\prime}+\bar{a} \bar{a}^{\prime}+\bar{a}^{\prime} \bar{b}^{\prime}+\bar{c}^{\prime}=\bar{a}_{v}+\bar{a} \bar{a}^{\prime}+\bar{b} \bar{a}^{\prime \prime},
$$

etc. There is no theoretical difficulty in the calculation of these coefficients, which we leave for the present.

Let $P_{\bar{y}}$ be any point of the plane. We may suppose without loss of generality that for $P_{\bar{y}}$ the parameters $\bar{u}, \bar{v}$ have the values 0,0 . Assume that about $P_{\bar{y}}$ the geometric configuration is regular, so that for a sufficiently small neighborhood about $P_{\bar{y}}$ we have the expansion

$$
\mathrm{Y}=\bar{y}+\bar{y}_{u} \bar{u}+\bar{y}_{v} \bar{v}+\frac{1}{2}\left(\bar{y}_{u u} \bar{u}^{2}+2 \bar{y}_{u v} \bar{u} \bar{v}+\bar{y}_{v v} \bar{v}^{2}\right)+\cdots,
$$

where $\mathrm{Y}$ corresponds to general values of the parameters $\bar{u}, \bar{v}$. By using equations (15) we find, therefore,

$$
\mathrm{Y}=y_{1} \bar{\rho}+y_{2} \bar{\sigma}+y_{3} \bar{y}
$$

where $y_{1}, y_{2}, y_{3}$ are the following power series in $\bar{u}, \bar{v}$ :

* The equality of the pairs of expressions which represent the six coefficients $\alpha^{(21)}, \beta^{(21)}$, $\gamma^{(21)}, \alpha^{(12)}, \beta^{(12)}, \gamma^{(12)}$ is sufficient to ensure the equality of ambiguous expressions for all other coefficients. In fact, the six equations expressing the uniqueness in value of $\alpha^{(21)}, \beta^{(21)}$, etc., are the conditions of complete integrability for the system of differential equations (15b). 


$$
\begin{aligned}
y_{1}= & \bar{u}+\frac{1}{2} \bar{a} \bar{u}^{2}+\bar{a}^{\prime} \bar{u} \bar{v}+\frac{1}{2} \bar{a}^{\prime \prime} \bar{v}^{2} \\
& +\frac{1}{6} \alpha^{(30)} \bar{u}^{3}+\frac{1}{2} \alpha^{(21)} \bar{u}^{2} \bar{v}+\frac{1}{2} \alpha^{(12)} \bar{u} \bar{v}^{2}+\frac{1}{6} \alpha^{(03)} \bar{v}^{(3)} \\
& +\frac{1}{2} \alpha^{(40)} \bar{u}^{4}+\frac{1}{6} \alpha^{(31)} \bar{u}^{3} \bar{v}+\frac{1}{4} \alpha^{(22)} \bar{u}^{2} \bar{v}^{2}+\frac{1}{6} \alpha^{(13)} \bar{u} \bar{v}^{8}+\frac{1}{2} \alpha^{(04)} \bar{v}^{4} \\
& +\cdots, \\
y_{2}= & \bar{v}+\frac{1}{2} \bar{b} \bar{u}^{2}+\bar{b}^{\prime} \bar{u} \bar{v}+\frac{1}{2} \bar{b}^{\prime \prime} \vec{v}^{2} \\
& +\frac{1}{6} \beta^{(30)} \bar{u}^{3}+\frac{1}{2} \beta^{(21)} \bar{u}^{2} \bar{v}+\frac{1}{2} \beta^{(12)} \bar{u} \bar{v}^{2}+\frac{1}{6} \beta^{(03)} \vec{v}^{3} \\
& +\frac{1}{2} \beta^{(40)} \bar{u}^{4}+\frac{1}{6} \beta^{(31)} \bar{u}^{3} \bar{v}+\frac{1}{4} \beta^{(22)} \bar{u}^{2} \bar{v}^{2}+\frac{1}{6} \beta^{(13)} \bar{u} \vec{v}^{3} \\
& +\frac{1}{2} \bar{s}^{(04)} \bar{v}^{4}+\cdots, \\
y_{3}= & +\bar{b}^{\prime} \bar{u}+\bar{a}^{\prime} \bar{v}+\frac{1}{2} \gamma^{(20)} \bar{u}^{2}+\gamma^{(11)} \bar{u} \bar{v}+\frac{1}{2} \gamma^{(02)} \vec{v}^{2} \\
& +\frac{1}{6} \gamma^{(30)} \bar{u}^{3}+\frac{1}{2} \gamma^{(21)} \bar{u}^{2} \bar{v}+\frac{1}{2} \gamma^{(12)} \bar{u} \bar{v}^{2}+\frac{1}{6} \gamma^{(03)} \bar{v}^{8} \\
& +\cdots,
\end{aligned}
$$

in which terms omitted are in each case of higher order than the last term written.

Except for isolated points $\bar{y}$ of the plane, the covariant points $\bar{\rho}, \bar{\sigma}$ which correspond to the point $\bar{y}$ form with $\bar{y}$ a non-degenerate triangle, which we shall take as a moving triangle of reference. A point whose three coördinates are

$$
x^{(k)}=x_{1} \bar{\rho}^{(k)}+x_{2} \bar{\sigma}^{(k)}+x_{3} \bar{y}^{(k)} \quad(k=1,2,3),
$$

or, as we may say, a point given by

$$
x=x_{1} \bar{\rho}+x_{2} \bar{\sigma}+x_{3} \bar{y},
$$

will have coördinates $x_{1}, x_{2}, x_{3}$ when referred to the covariant triangle $P_{\rho}$, $P_{\bar{\sigma}}, P_{\bar{y}}$, if the unit point of the new coördinate system be properly chosen. Hence the point $Y$, given by (17), has coördinates $y_{1}, y_{2}, y_{3}$ when referred to the triangle $P_{\bar{p}}, P_{\bar{\sigma}}, P_{\bar{y}}$.

We now introduce non-homogeneous coördinates $\xi$, $\eta$, by putting

$$
\xi=\frac{y_{1}}{y_{8}}, \quad \eta=\frac{y_{2}}{y_{8}} .
$$

We have, to terms of the third order,

$$
\begin{aligned}
\frac{1}{y_{3}}=1-\bar{b}^{\prime} \bar{u}-\bar{a}^{\prime} \bar{v}+C^{(20)} & \bar{u}^{2}+C^{(11)} \bar{u} \bar{v}+C^{(02)} \vec{v}^{2} \\
& +C^{(30)} \bar{u}^{3}+C^{(21)} \bar{u}^{2} \bar{v}+C^{(12)} \bar{u} \bar{v}^{2}+C^{(03)} \vec{v}^{3}+\cdots,
\end{aligned}
$$


where

$$
\begin{aligned}
& C^{(20)}=\bar{b}^{\prime 2}-\frac{1}{2} \gamma^{(20)}, \quad C^{(11) \cdot}=2 \bar{a}^{\prime} \bar{b}^{\prime}-\gamma^{(11)}, \quad C^{(02)}=\bar{a}^{\prime 2}-\frac{1}{2} \gamma^{(02)}, \\
& C^{(30)}=-\bar{b}^{\prime 3}+\bar{b}^{\prime} \gamma^{(20)}-\frac{1}{6} \gamma^{(30)} \\
& C^{(21)}=-3 \bar{a}^{\prime} \bar{b}^{\prime 2}+\bar{a}^{\prime} \gamma^{(20)}+2 \bar{b}^{\prime} \gamma^{(11)}-\frac{1}{2} \gamma^{(21)}, \\
& C^{(12)}=-3 \bar{a}^{\prime 2} \bar{b}^{\prime}+\bar{b}^{\prime} \gamma^{(02)}+2 \bar{a}^{\prime} \gamma^{(11)}-\frac{1}{2} \gamma^{(12)}, \\
& C^{(03)}=-\bar{a}^{\prime 3}+\bar{a}^{\prime} \gamma^{(02)}-\frac{1}{6} \gamma^{(03)} .
\end{aligned}
$$

We therefore obtain for $\xi$ and $\eta$ the following expressions, the one for $\xi$ being exact to terms of the fourth order, and the one for $\eta$ being calculated only to terms of the third order.

$$
\begin{aligned}
\xi= & \bar{u}+\left(\frac{1}{2} \bar{a}-\bar{b}^{\prime}\right) \bar{u}^{2}+\frac{1}{2} \bar{a}^{\prime \prime} \bar{v}^{2} \\
& +A^{(30)} \bar{u}^{3}+A^{(21)} \bar{u}^{2} \bar{v}+A^{(12)} \bar{u} \bar{v}^{2}+A^{(03)} \bar{v}^{3} \\
& +A^{(40)} \bar{u}^{4}+A^{(31)} \bar{u}^{3} \bar{v}+A^{(22)} \bar{u}^{2} \bar{v}^{2}+A^{(13)} \bar{u} \bar{v}^{3}+A^{(04)} \bar{v}^{4}+\cdots, \\
\eta= & \bar{v} \frac{1}{2} \bar{b} \bar{u}^{2}+\left(\frac{1}{2} \bar{b}^{\prime \prime}-\bar{a}^{\prime}\right) \bar{v}^{2} \\
& +B^{(30)} \bar{u}^{3}+B^{(21)} \bar{u}^{2} \bar{v}+B^{(12)} \bar{u} \bar{v}^{2}+B^{(03)} \bar{v}^{3}+\cdots,
\end{aligned}
$$

where$$
\begin{aligned}
& A^{(30)}=\frac{1}{6} \alpha^{(30)}-\frac{1}{2} \bar{a} \bar{b}^{\prime}+C^{(20)}, \quad A^{(21)}=\frac{1}{2} \alpha^{(21)}-\bar{a}^{\prime} \bar{b}^{\prime}-\frac{1}{2} \bar{a} \bar{a}^{\prime}+C^{(11)}, \\
& A^{(12)}=\frac{1}{2} \alpha^{(12)}-\frac{1}{2} \bar{a}^{\prime \prime} \bar{b}^{\prime}-\bar{a}^{\prime 2}+C^{(02)}, \quad A^{(03)}=\frac{1}{6} \alpha^{(03)}-\frac{1}{2} \bar{a}^{\prime} \bar{a}^{\prime \prime}, \\
& A^{(40)}=\frac{1}{2} \alpha^{(40)}-\frac{1}{6} \bar{b}^{\prime} \alpha^{(30)}+\frac{1}{2} \bar{a} C^{(20)}+C^{(30)}, \\
& A^{(31)}=\frac{1}{6} \alpha^{(31)}-\frac{1}{2} \bar{b}^{\prime} \alpha^{(21)}-\frac{1}{6} \bar{a}^{\prime} \alpha^{(30)}+\bar{a}^{\prime} C^{(20)}+\frac{1}{2} \bar{a} C^{(11)}+C^{(21)}, \\
& A^{(22)}=\frac{1}{2} \alpha^{(22)}-\frac{1}{2} \bar{b}^{\prime} \alpha^{(12)}-\frac{1}{2} \bar{a}^{\prime} \alpha^{(21)}+\frac{1}{2} \bar{a}^{\prime \prime} C^{(20)}+\bar{a}^{\prime} C^{(11)}
\end{aligned}
$$

$$
\begin{aligned}
& A^{(13)}=\frac{1}{6} \alpha^{(13)}-\frac{1}{6} \bar{b}^{\prime} \alpha^{(03)}-\frac{1}{2} \bar{a} C^{(02)}+C^{(12)}, \\
& A^{(04)}=\frac{1}{2} \alpha^{(12)}+\frac{1}{2} \bar{a}^{\prime \prime} C^{(11)}+\frac{1}{6} \bar{a}^{\prime} \alpha^{(03)}+\frac{1}{2} \bar{a}^{\prime \prime} C^{(02)}+C^{(03)}, \\
& B^{(30)}=\frac{1}{6} \beta^{(20)}-\frac{1}{2} \bar{b} \bar{b}^{\prime}, \quad B^{(21)}=\frac{1}{2} \beta^{(21)}-\bar{b}^{\prime 2}-\frac{1}{2} \bar{a}^{\prime} \bar{b}+C^{(20)}, \\
& B^{(12)}=\frac{1}{2} \beta^{(12)}-\frac{1}{2} \bar{b}^{\prime} \bar{b}^{\prime \prime}-\bar{a}^{\prime} \bar{b}^{\prime}+C^{(11)}, \\
& B^{(03)}=\frac{1}{6} \beta^{(03)}-\frac{1}{2} \bar{a}^{\prime} \bar{b}^{\prime \prime}+C^{(02)} .
\end{aligned}
$$

We wish to express $\xi$ as a power series in $\bar{u}$ and $\eta$. We have from the second of (20), to terms of the fourth order,*

* Our subsequent developments are valid even though the last term in the second of equations (20) is of the third order. 


$$
\begin{aligned}
\eta^{4}=\bar{v}^{4}, \quad \eta^{3} & =\bar{v}^{3}+\frac{8}{2} \bar{b} \bar{u}^{2} \bar{v}^{2}+\frac{3}{2}\left(\bar{b}^{\prime \prime}-2 \bar{a}^{\prime}\right) \bar{v}^{4} \\
\eta^{2}=\vec{v}^{2}+b \bar{u} \bar{u}^{2} \bar{v} & +\left(\bar{b}^{\prime \prime}-2 \bar{a}^{\prime}\right) \bar{v}^{3}+\frac{1}{4} \bar{b}^{2} \bar{u}^{4}+2 B^{(30)} \bar{u}^{3} \bar{v} \\
& +\left[2 B^{(21)}+\frac{1}{2} \bar{b}\left(\bar{b}^{\prime \prime}-2 \bar{a}^{\prime}\right)\right] \bar{u}^{2} \vec{v}^{2}+2 B^{(12)} \bar{u} \vec{v}^{3} \\
& +\left[2 B^{(03)}+\frac{1}{4}\left(\bar{b}^{\prime \prime}-2 \bar{a}^{\prime}\right)^{2}\right] \bar{v}^{4} .
\end{aligned}
$$

We have therefore, exact to terms of the fourth order,

$$
\begin{aligned}
\bar{v}^{4} & =\eta^{4}, \quad \bar{u} \bar{v}^{3}=\bar{u} \eta^{3}, \quad \bar{u}^{2} \bar{v}^{2}=\bar{u}^{2} \eta^{2}, \quad \bar{u}^{3} \bar{v}=\bar{u}^{3} \eta \\
\bar{v}^{3} & =\eta^{3}-\frac{8}{2} \bar{b} \bar{u}^{2} \eta^{2}-\frac{3}{2}\left(\bar{b}^{\prime \prime}-2 \bar{a}^{\prime}\right) \eta^{4}, \\
\bar{u} \bar{v}^{2} & =\bar{u} \eta^{2}-\bar{b} \bar{u}^{3} \eta-\left(\bar{b}^{\prime \prime}-2 \bar{a}^{\prime}\right) \bar{u} \eta^{3}, \\
\bar{u}^{2} \bar{v}= & \bar{u}^{2} \eta-\frac{1}{2} \bar{b} \bar{u}^{4}-\frac{1}{2}\left(\bar{b}^{\prime \prime}-2 \bar{a}^{\prime}\right) \bar{u}^{2} \eta^{2}, \\
\bar{v}^{2}= & \eta^{2}-\bar{b} \bar{u}^{2} \eta-\left(\bar{b}^{\prime \prime}-2 \bar{a}^{\prime}\right) \eta^{3}+\frac{1}{4} \bar{b}^{2} \bar{u}^{4}-2 B^{(30)} \bar{u}^{3} \eta \\
& \quad+\left[\frac{8}{2} \bar{b}\left(\bar{b}^{\prime \prime}-2 \bar{a}^{\prime}\right)-2 B^{(21)}\right] \bar{u}^{2} \eta^{2}-2 B^{(12)} \bar{u} \eta^{3} \\
& \quad+\left[\frac{5}{4}\left(\bar{b}^{\prime \prime}-2 \bar{a}^{\prime}\right)^{2}-2 B^{(03)}\right] \eta^{4} .
\end{aligned}
$$

Substituting these in the first of equations (20), we obtain

$$
\begin{aligned}
\xi=\bar{u} & +\frac{1}{2}\left(\bar{a}-2 \bar{b}^{\prime}\right) \bar{u}^{2}+A^{(30)} \bar{u}^{3}+\left[A^{(40)}-\frac{1}{2} \bar{b} A^{(21)}+\frac{1}{8} \bar{a}^{\prime \prime} \bar{b}^{2}\right] \bar{u}^{4} \\
& +\cdots+\frac{1}{2} \bar{a}^{\prime \prime} \eta^{2}+\left[A^{(03)}-\frac{1}{2} \bar{a}^{\prime \prime}\left(\bar{b}^{\prime \prime}-2 \bar{a}^{\prime}\right)\right] \eta^{3} \\
& +\left[A^{(04)}-\frac{8}{2}\left(\bar{b}^{\prime \prime}-2 \bar{a}^{\prime}\right) A^{(03)}+\frac{5}{8} \bar{a}^{\prime \prime}\left(\bar{b}^{\prime \prime}-2 \bar{a}^{\prime}\right)^{2}-\bar{a}^{\prime \prime} B^{(03)}\right] \eta^{4} \\
& +\cdots+\left\{\left(A^{(21)}-\frac{1}{2} \bar{a}^{\prime \prime} \bar{b}\right) \bar{u}^{2}+\left(A^{(31)}-\bar{b} A^{(12)}-\bar{a}^{\prime \prime} B^{(30)}\right) \bar{u}^{3}\right. \\
& +\cdots\} \eta+\left\{A^{(12)} \bar{u}+\left[A^{(22)}-\frac{3}{2} \bar{b} A^{(03)}-\frac{1}{2}\left(\bar{b}^{\prime \prime}-2 \bar{a}^{\prime}\right) A^{(21)}\right.\right. \\
& \left.\left.-\bar{a}^{\prime \prime} B^{(21)}+\frac{3}{4} \bar{a}^{\prime \prime} \bar{b}\left(\bar{b}^{\prime \prime}-2 \bar{a}^{\prime}\right)\right] \bar{u}^{2}+\cdots\right\} \eta^{2} \\
& +\left\{\left[A^{(13)}-\left(\bar{b}^{\prime \prime}-2 \bar{a}^{\prime}\right) A^{(12)}-\bar{a}^{\prime \prime} B^{(12)}\right] \bar{u}+\cdots\right\} \eta^{3} \\
& +\cdots+\cdots
\end{aligned}
$$

We have here arranged the expression for $\xi$ in three parts. The first part is a power series in $\bar{u}$ alone, the second part is a power series in $\eta$ alone, and the third part is a power series in $\eta$ whose coefficients are power series in $\bar{u}$.

Since the triangle of reference is a covariant triangle, we might expect the coefficients in the development to be invariants; but not all of them are, because the parameter $\bar{u}$ is not chosen in any unique way. In fact, in the part containing powers of $\bar{u}$ alone, none of the coefficients is an invariant, while in the part containing powers of $\eta$ alone all of the coefficients are invariants. In particular, the coefficient of $\eta^{3}$ vanishes identically:

$$
A^{(03)}-\frac{1}{2} \bar{a}^{\prime \prime}\left(\bar{b}^{\prime \prime}-2 \bar{a}^{\prime}\right)=0 \text {. }
$$


Again, in the part which contains powers of both $\bar{u}$ and $\eta$, we have enclosed the coefficient of each power of $\eta$ in a brace \{\} . Only the first coefficient in each brace is an invariant. Thus, in the term

$$
\left\{\left(A^{(21)}-\frac{1}{2} \bar{a}^{\prime \prime} \bar{b}\right) \bar{u}^{2}+\left(A^{(31)}-\bar{b} A^{(12)}-\bar{a}^{\prime \prime} B^{(30)}\right) \bar{u}^{3}+\cdots\right\} \eta,
$$

the quantity $A^{(21)}-\frac{1}{2} \bar{a}^{\prime \prime} \bar{b}$ is an invariant, while $A^{(21)}-\bar{b} A^{(12)}-\bar{a}^{\prime \prime} B^{(30)}$ is not. In fact, following out the notation of Wilczynski, we may verify that

$$
\begin{aligned}
& A^{(21)}-\frac{1}{2} \bar{a}^{\prime \prime} \bar{b}=-\frac{1}{2}\left(\bar{H}+\overline{\mathfrak{A}^{\prime \prime}} \bar{B}\right) \text {, } \\
& A^{(31)}-\bar{b} A^{(12)}-\bar{a}^{\prime \prime} B^{(20)}=-\frac{1}{2} \overline{\mathfrak{B}}\left(\overline{\mathfrak{A}}^{\prime \prime}, \bar{B}^{\prime}\right)-\overline{\mathfrak{A}^{\prime \prime}} \overline{\mathfrak{B}} \overline{\mathfrak{B}}^{\prime}-\frac{1}{6}\left(\bar{H}, \bar{B}^{\prime}\right) \\
& -\frac{1}{2}\left(\bar{a}-2 \bar{b}^{\prime}\right)\left(\bar{H}+\overline{\mathfrak{Q}}^{\prime \prime} \overline{\mathfrak{B}}\right) \text {, }
\end{aligned}
$$

where

$$
\begin{gathered}
\bar{H}=\bar{c}^{\prime}+\bar{a}^{\prime} \bar{b}^{\prime}-\bar{a}_{u}^{\prime}, \quad \overline{\mathfrak{A}}^{\prime \prime}=\bar{a}^{\prime \prime}, \quad \overline{\mathfrak{B}}=\bar{b}, \quad \overline{\mathfrak{B}}^{\prime}=\frac{1}{3}\left(2 \bar{b}^{\prime}-\bar{a}\right)+\frac{1}{6} \frac{\bar{b}_{u}}{\bar{b}}, \\
\left(\overline{\mathfrak{A}}^{\prime \prime}, \bar{B}^{\prime}\right)=\overline{\mathfrak{A}}_{u}^{\prime \prime}-\bar{B}^{\prime} \mathfrak{A}^{\prime \prime}=\bar{a}_{u}^{\prime \prime}+\left(\bar{a}-2 \bar{b}^{\prime}\right) \bar{a}^{\prime \prime}, \\
\left(\bar{H}, \bar{B}^{\prime}\right)=\bar{H}_{u}+\bar{B}^{\prime} \bar{H}=\bar{H}_{u}-\left(\bar{a}-2 \bar{b}^{\prime}\right) \bar{H}
\end{gathered}
$$

are invariants of the canonical net. Then the second of (25) is not invariant, since the quantity $\bar{a}-2 \bar{b}^{\prime}$ is not.

If instead of the parameter $\bar{u}$ we choose a uniquely determined parameter $\tau$, it will be possible to transform (22) into a new expansion in which all the coefficients are relative invariants. This may be done by taking for $\tau$ the part of (22) containing powers of $\bar{u}$ alone, i. e.,

$$
\tau=\bar{u}+\frac{1}{2}\left(\bar{a}-2 \bar{b}^{\prime}\right) \bar{u}^{2}+A^{(30)} \bar{u}^{3}+\cdots,
$$

the inversion of which gives

$$
\begin{aligned}
\bar{u} & =\tau-\frac{1}{2}\left(\bar{a}-2 \bar{b}^{\prime}\right) \tau^{2}+\cdots, \\
\bar{u}^{2} & =\tau^{2}-\left(\bar{a}-2 \bar{b}^{\prime}\right) \tau^{3}+\cdots,
\end{aligned}
$$

Each brace in (22) becomes a new power series in $\tau$, with invariants as coefficients; thus, in particular, the term (24) becomes

$$
\left\{-\frac{1}{2}\left(\bar{H}+\overline{\mathfrak{Q}^{\prime \prime}} \overline{\mathfrak{B}}\right) \tau^{2}-\left[\frac{1}{2} \overline{\mathscr{B}}\left(\overline{\mathfrak{A}^{\prime \prime}}, \bar{B}^{\prime}\right)+\overline{\mathfrak{X}^{\prime \prime}} \overline{\mathfrak{B}} \overline{\mathfrak{B}^{\prime}}-\frac{1}{6}\left(\bar{H}, \bar{B}^{\prime}\right)\right] \tau^{3}+\cdots\right\} \eta \text {. }
$$

The coefficient of $\eta^{4}$ in (22) is found without much difficulty to have the value

$$
-\frac{\overline{\mathfrak{P}^{\prime \prime}}}{8}\left(\overline{\mathfrak{A}}^{\prime \prime}, \bar{B}^{\prime}\right) \text {, }
$$

so that we have the development 


$$
\begin{aligned}
\xi=\tau & +\frac{1}{2} \bar{a}^{\prime \prime} \eta^{2}-\frac{1}{8} \bar{a}^{\prime \prime}\left(\overline{\mathfrak{U}^{\prime \prime}}, \bar{B}^{\prime}\right) \eta^{4}+\cdots \\
& +\left\{\theta^{(21)} \tau^{2}+\theta^{(31)} \tau^{3}+\cdots\right\} \eta \\
& +\left\{\theta^{(12)} \tau+\theta^{(22)} \tau^{2}+\cdots\right\} \eta^{2} \\
& +\left\{\theta^{(13)} \tau+\cdots\right\} \eta^{3}+\cdots,
\end{aligned}
$$

where $\theta^{(i j)}$, the coefficient of $\tau^{i} \eta^{j}$, is a relative invariant of the canonical net.

We may make some further transformations, which will yield a development with absolute invariants as coefficients. The coefficients in (26) are of course constants, being the values of the corresponding invariants for the values 0,0 of the arguments $\bar{u}, \bar{v}$. Consider the invariant $\theta^{(i j)}$; it is easily verified that the point transformation

changes it into

$$
\bar{u}=U(\bar{u}), \quad \bar{v}=V(\bar{v})
$$

$$
\bar{\theta}^{(i j)}=\frac{1}{\left(U^{\prime}\right)^{i-1}} \cdot \frac{1}{\left(V^{\prime}\right)^{j}} \theta^{(i j)},
$$

where $U^{\prime}$ and $V^{\prime}$ are the first derivatives of $U$ and $V$. The same transformation changes the invariants $\bar{a}^{\prime \prime}$ and $\bar{b}$ into

Therefore

$$
\bar{a}^{\prime \prime}=\frac{U^{\prime}}{V^{\prime 2}} \bar{a}^{\prime \prime}, \quad \bar{b}=\frac{V^{\prime}}{U^{\prime 2}} \bar{b} .
$$

Let us put

Then the quantity

$$
\bar{a}^{\prime \prime} \bar{b}^{2}=\frac{1}{U^{\prime^{3}}} \bar{a}^{\prime \prime} \bar{b}^{2}, \quad \bar{a}^{\prime \prime 2} \bar{b}=\frac{1}{V^{\prime^{3}}} \bar{a}^{\prime \prime 2} \bar{b}
$$

$$
\mu=\sqrt[3]{\overline{\bar{a}^{\prime \prime} \bar{b}^{2}}}, \quad \nu=\sqrt[3]{\overline{\bar{a}^{\prime \prime 2} \bar{b}}} .
$$

$$
I^{(i j)}=\mu \cdot\left(\frac{1}{\mu}\right)^{i}\left(\frac{1}{\nu}\right)^{j} \theta^{(i j)}
$$

is an absolute invariant. In fact, the transformed quantity is seen from (27) and (28) to satisfy the equation

$$
\bar{I}^{(i j)}=I^{(i j)} .
$$

If, then, in the expansion (26) we put*

$$
x=\mu \xi, \quad y=\nu \eta, \quad t=\mu \tau,
$$

we obtain the final canonical development,

$$
\begin{aligned}
x=t & +\frac{1}{2} y^{2}+I^{(04)} y^{4}+\cdots \\
& +\left\{I^{(21)} t^{2}+I^{(31)} t^{3}+\cdots\right\} y \\
& +\left\{I^{(12)} t+I^{(22)} t^{2}+\cdots\right\} y^{2} \\
& +\left\{I^{(13)} t+\cdots\right\} y^{3}+\cdots,
\end{aligned}
$$

the coefficients of which are all absolute invariants.

* The transformation (31) leaves fixed the covariant triangle $P_{\bar{y}}, P_{\bar{p}}, P_{\bar{\sigma}}$. 
In recapitulation, we state the result:

$A$ one-parameter family of analytic plane curves, given by the equations

$$
y^{(k)}=f^{(k)}(u, v) \quad(k=1,2,3)
$$

for $u=$ const., may be represented in the neighborhood of a regular point by the canonical development (31) for $t=$ const. In this development all the coefficients are absolute projective invariants. The triangle of reference which gives rise to this development is the covariant triangle $P_{\bar{y}}, P_{\bar{\rho}}, P_{\bar{\sigma}}$, of which a purely geometric interpretation has been given. The parameter $t$ is uniquely determined as an analytic function of the old parameter $u$.

In the derivation of this canonical development, the essential steps to be noted are first, the determination of a triangle of reference which bears a covariant relation to the one-parameter family of curves (considered apart from any net of which it may be a component family), and secondly the characteristic choice of the parameter $t$. From the results stated in $\$ 1$, we see that the absolute invariants which enter into the canonical development as coefficients are all expressible rationally in terms of the coefficients of the original differential equations which define the family of curves as a component of a net in which the second family bears only an accidental relation to the given family. Our canonical development is therefore independent of the integration which is necessary to determine the canonical net.

College of the City of New York,

October, 1913. 